\title{
Effects of a Mental Health Carve-Out on Use, Costs, and Payers: A Four-Year Study
}

\author{
Kyle L. Grazier, Dr.P.H. \\ Laura L. Eselius, M.P.H. \\ Teh-wei Hu, Ph.D. \\ Karen K. Shore, Ph.D. \\ William A. G'Sell, M.S., J.D.
}

\begin{abstract}
This study examines the effects of a mental health carve-out on a sample of continuously enrolled employees $(\mathrm{N}=1,943)$ over a four-year time frame (1990-1994). The article presents a health care services utilization model of the effect of the carve-out on outpatient mental health use, cost, and source of payment in the three years post implementation relative to the year prior to the carve-out model. In the first three years of the carve-out, the likelihood of employees seeking mental health care increased in significant part because of the carve-out. For the outpatient mental health services user, the carve-out was not associated with the level of mental health services received. The carveout was significantly associated over time with a reduction in the patient's and employer's mental health costs. This effect was more pronounced in the second and third years of the carve-out. The article explores the policy implications of these and other findings.
\end{abstract}

An increasingly common approach to containing mental health costs and improving access to mental health services is a carve-out strategy, whereby mental health and substance abuse services are separated from other types of health care services and managed by a specialty vendor who may assume some level of financial risk. Carve-out arrangements are occurring at different levels of the health care system; payers (both public and private), health plans (both indemnity and managed care), and even group practices are contracting with specialty organizations to manage a segment of their insurance risk for these services.'

These arrangements vary in many respects, including general form (e.g., carve-out from all health plans or from only indemnity and preferred provider organization-PPO-plans), benefit design, provider network characteristics and fee arrangements, vendor characteristics and management techniques, contract features, enrollee characteristics, and market context. ${ }^{1.2}$ Research into the effects of carve-outs of different types is critical for building an understanding of which features influence costs and utilization, in what ways, and for which populations.

Address correspondence to Kyle L. Grazier, Dr.P.H., Associate Professor, School of Public Health, 109 South Observatory, University of Michigan, Ann Arbor, MI 48109-2029; e-mail: KGrazier@umich.edu.

Laura L. Eselius, M.P.H., is a doctoral candidate at Harvard University, Boston.

Teh-wei Hu, Ph.D., is professor of health economics in the School of Public Health, University of California, Berkeley.

Karen K. Shore, Ph.D, is with Reden \& Anders Ltd. in San Francisco.

William A. G'Sell, M.S., J.D., is a senior research investigator at the University of Michigan, Ann Arbor. 
Numerous reports and studies from both the private and public sector suggest that a carve-out strategy can reduce total costs for mental health and substance abuse services, ${ }^{2-9}$ even when the number of service users increases. ${ }^{4-6}$ Significant cost reductions have been seen in carve-outs of different types involving no risk sharing, ${ }^{4}$ some risk sharing, ${ }^{5-8}$ and full risk sharing. ${ }^{9}$ In many cases, sizable declines (17\% to $42 \%$ ) in total or average expenditures for mental health and substance abuse services managed by the vendor have occurred in the first year, followed by smaller declines in subsequent years. ${ }^{4.8}$ Cost savings among subgroups of enrollees who are severely mentally ill and disabled have been even higher (reductions of $33 \%$ to $53 \%$ ), suggesting that managed mental health and substance abuse services achieve the greatest savings from those who are sickest. ${ }^{5,10-12}$

Cost savings seem largely due to decreases in inpatient services and, more specifically, to reductions in admissions, $4,5,8,10,12-14$ total number of inpatient days (measured as a rate per 1,000 enrollees), ${ }^{9,15}$ lengths of stay, ${ }^{4,5,10,13.15}$ and per diem costs or reimbursement rates. ${ }^{4,5,7.10}$ In the one reported case where an increase was seen in inpatient services (in the probability of admission), overall declines in inpatient expenditures still resulted from reductions in the intensity of inpatient services (inpatient days per 1,000 and length of stay) and expenditures per admission. ${ }^{7,15}$

While the findings regarding inpatient services have been quite similar across studies, the effects of carve-outs on outpatient costs and utilization have varied. For instance, outpatient costs declined in the first year of two carve-outs (13\% to $20 \%)^{5,15}$ but remained about the same in another. ${ }^{8,14}$ When cost reductions have occurred, they have occurred both when the number or proportion of users of outpatient services has increased, ${ }^{5}$ as well as when the number or proportion of users has decreased. ${ }^{7.15}$ Costs appear to decline in these cases because of reductions in the number of outpatient visits per user, ${ }^{4,7,16}$ and the costs per session. ${ }^{4,5,7,15}$

Generally, the mix of services used appears to shift; some inpatient care is replaced by less costly intermediate care (e.g., residential services) and outpatient care. Evidence of cost shifting among service providers (e.g., hospitals) and service sectors (e.g., medical services and pharmacy) does exist. ${ }^{10-13}$ However, the one study we found that examines potential cost shifting from the payer (an employer) to the enrollees (employees) showed no evidence of this effect. ${ }^{7}$

This article presents the results of a four-year study of a private sector carve-out based on an administrative-services-only (no risk sharing) contract. We focus on the effect of the carve-out over time on the probability and intensity of use of outpatient mental health services and the costs to the employee and employer. The carve-out is examined in the context of an expansion of mental health benefits for 1,943 enrollees exposed to the carve-out option.

\section{Carve-Out Case Description}

In January 1991, a national self-funded employer implemented a mental health carve-out for employees and dependents covered by its PPO plan. The carve-out firm has an administrativeservices-only contract with the employer to provide and manage inpatient and outpatient mental health services. The employer pays the vendor a monthly fee per enrollee for the administration of claims and care management but retains all financial risk for the claims. The vendor offers contracts to providers who are located in catchment areas where employees live and work, who are licensed, and who are willing to comply with the vendor's rules for the monitoring and approval of services. The network of practitioners on contract with the vendor includes psychiatrists, psychologists, and master's-level, independently licensed therapists (e.g., social workers and family counselors). Providers are not financially at risk but agree to provide services at a negotiated rate.

Although the provider network has changed little since the inception of the plan, the process of managing treatment changed at one point for outpatient care. During the first year of the carve-out, providers were allowed to see the client for 10 visits prior to filing a treatment plan report with the vendor, after which the vendor certified the need for additional therapy, up to 30 visits in a contract year. In the second year, 1992, the policy was made somewhat more burdensome in that the vendor 
required a treatment plan report after the fifth visit and again after every three visits. There were no changes to the inpatient case management process over the study years; a treatment plan report was required prior to admission and again after every three days of stay.

Employees who sought mental health care could choose upon each visit either to use a network provider or a non-network provider. There were financial incentives to encourage enrollees to use network providers. For network outpatient mental health services, employees faced a copayment of $\$ 10$ per visit for the first 10 visits, and $\$ 30$ per visit for visits 11 through the maximum of 30 visits allowed in the contract year. The plan covered the balance of the visit charges up to a maximum of $\$ 3,000$ per calendar year. However, for non-network outpatient care, the plan imposed a $\$ 400$ deductible (a joint deductible for medical and mental health use), after which the plan paid $30 \%$ of the reasonable and customary costs of covered services up to a maximum of $\$ 1,500$ per contract year. Similarly, if the employee sought inpatient mental health care through the vendor, it required approval; the plan paid $90 \%$ of the eligible charges for up to 45 days of hospitalization in any 12month period. Non-network inpatient care was only available in an emergency and only after authorization; after the deductible, the plan covered $50 \%$ of reasonable and customary charges for the authorized emergency care, limited to 30 days per 12-month period.

Employees who seek care for substance abuse services have the option of using the on-site employee assistance program (EAP). Licensed counselors can diagnose, treat, and monitor treatment for employees and their dependents; the program does not specify a maximum length of service. This employer does not release records of use of EAP, and all related data are confidential. For this reason, we are unable to capture the probability of use of substance abuse services or the level of use and costs that might be associated with EAP and/or the carve-out. We therefore exclude examination of the effect of the carve-out on use and costs of substance abuse services. The study seeks only to analyze the carve-out's impact on cost and use of mental health services.

\section{Plan Features: Preintervention}

In 1990 , the year prior to implementation and after a $\$ 400$ combined mental health $/$ medical deductible, the plan paid $80 \%$ of eligible outpatient mental health service expenses, limited to $\$ 1,500$ per person per calendar year. The inpatient mental health benefit provided 45 hospital days in any 12 -month period. Throughout the study period, the lifetime maximum amount for mental health benefits was $\$ 100,000$ for each covered person. For medical care, after a $\$ 400$ per person deductible, the plan paid $90 \%$ of negotiated charges for care received from preferred providers and $80 \%$ of covered expenses for care in which non-preferred providers were used. A $\$ 3,000$ per person out-ofpocket limit was in place for medical care, after which the plan paid $100 \%$ of eligible expenses. The lifetime maximum benefit for medical expenses was $\$ 1$ million.

\section{Model Conceptualization and Empirical Specification}

The study employs a health care services utilization model for mental health care in an employed population that had experienced a change in mental health coverage over time through the introduction of a carve-out. Because inpatient use did not vary significantly over time, and due to very small numbers of inpatient cases, the sample for analysis excludes users of inpatient services.*

The model assumes that use and cost of outpatient mental health services is a function of the employee's age, gender, race, number of dependents, marital status, salary, and length of employment with the firm. Demographic characteristics such as age, sex, race, and marital status have been

\footnotetext{
*Among the sample of continuously enrolled employees exposed to the preferred provider organization and point-ofservice plans, only a small number of employees filed inpatient mental health claims in any of the study years. There was no significant difference over time in the number of inpatient mental health cases.
} 
shown to affect utilization. The number of dependents, although not an accurate measure of family size, may reflect an income effect, as increased family size translates into fewer resources available for mental health services. However, a greater number of dependents can also mean a greater tendency to use outpatient services, as more complex interpersonal dynamics may cause a family member to seek mental health care. ${ }^{16}$ Tenure with an employer represents a degree of job stability; the longer someone is employed, the more likely the individual is able to carry out his or her job functions successfully. We expect that as length of employment increases, use of outpatient mental health services will decrease. ${ }^{16}$

In addition, we define three dummy variables, one for each year of the carve-out, so that changes over time in the effects of the carve-out can be compared and analyzed.

To examine the probability and level of mental health use and cost, we specify a two-stage model. A logit model estimates the probability of any outpatient mental health use. The second-stage model estimates the level of services or costs conditioned on any mental health outpatient utilization. Given that we have annual data on individuals continuously enrolled in the plan over a four-year period, we pooled individuals' data in our specified model for estimation. While this is an efficient approach to estimate the effect of a carve-out over time, pooling the same individuals over a four-year time period may lead to econometric challenges. For instance, high mental health services users may have larger variance in the error terms than those with lower use. Also, high-cost use by some individuals in the previous period may lead to high-cost use by the same individuals in the following period. These are so-called heteroscedasticity and autocorrelation problems that may both exist in the error term. When we assume that these individual effects and time effects are not correlated with other explanatory variables in the equations, it is called random effects; otherwise, it is called fixed effects. STATA's generalized estimating equation procedures are developed to estimate the random effects model; Hausman's test is used to test the null hypothesis of the existence of random effects versus the alternative hypothesis of fixed effects. When the estimated $\chi^{2}$ is less than the critical value, we do not reject the null hypothesis, indicating that the random effects model is an appropriate model to use. The unit of analysis is the person-year.

\section{Sample}

Only employees who were continuously employed and enrolled in the PPO/point-of-service (POS) plans over the four years of the study, and who therefore were continuously exposed to the plan, are examined here $(N=1,943)$. As noted earlier, this sample excludes those individuals who filed an inpatient claim with a primary mental health diagnosis. It also excludes those who enrolled in the available health maintenance organizations (HMOs), dependents, and any employees who left the company or switched plans (fewer than $3 \%$ in any one year). The sample may be a selected one; however, we do not estimate the bias resulting from any differences in HMO or indemnity plan enrollees prior to the introduction of the carve-out.

\section{Variable Measurement}

The employer, claims processors, and vendor provided data from personnel records, claims systems, and contract records for this analysis. The dependent variables consist of measures of mental health services utilization by the sample employees and costs incurred for services by the patients and by the employer. Eligibility and claims files provided utilization data for the study. We measure mental health use from claims records of visits or services with a primary diagnosis with an ICD-9 code from 290-302 or 306-315. We define mental health costs as the total payment in 1990 dollars for mental health services, the sum of the claim-specific deductibles, patient copayments, employer payment, and recovery from coordination of benefit collections.

Personnel, eligibility, provider, and claims files contribute data for the independent variables. Since income is not available, we use salary for the employee at the end of the year as noted in the firm's personnel files. Age at the end of the calendar year is computed from dates of birth recorded in 
the personnel files. It is parameterized at its actual value and square of its value. Personnel files provide date of hire, gender, and race (as self-reported on the employment application). The variable "years employed" is calculated from date of hire to the end of the study year. Salary (measured in $\$ 10,000$ increments), marital status, and number of dependents are recorded in annual personnel and eligibility files. A carve-out dummy variable $(0,1)$ is created for the absence or presence of the carve-out each year.

\section{Findings}

We present the characteristics of the sample of all employees and those who used outpatient mental health services, followed by the results from the modeling. Of the 1,943 full-time employees who were continuously employed and enrolled both before and after the carve-out was implemented (1990-1993), 64\% are female, $71 \%$ are white, and the mean age is 40 . The average length of employment is 10.4 years with a mean annual salary of approximately $\$ 41,500(1990)$. About $60 \%$ of the sample is married, with 1.06 dependents per employee (1990).

Over the four years of the study, the proportion of the sample using outpatient mental health services increased steadily until 1993 when there was a slight decrease. In 1990, the proportion of all employees using mental health services was .085 ; in the following three years, the proportions changed from .094 to .098 to $.091(p<.01)$.

The mean number of outpatient mental health visits per employee among the entire sample (users and nonusers) rose slightly in the first year of the carve-out (1.04 to 1.08) and then decreased significantly $(p<.05)$ each of the following two years $(1.06, .915)$. In the first year of the carve-out, the company's mean outpatient mental health service payment across all employees changed little from the previous year but then declined markedly and consistently in the following two years (\$55.4 in $1990, \$ 56.6$ in $1991, \$ 50.2$ in 1992 , and $\$ 40.6$ in $1993 ; p<.05$ ). The employee mean outpatient mental health services payment increased in the first year $(p<.05)$ but did not vary significantly over the years of the carve-out ( $\$ 24$ in 1990, $\$ 29$ in 1991, $\$ 30$ in 1992, and $\$ 28$ in 1993).

The sample of outpatient mental health services users $(n=167$ in 1990, 193 in 1991, 192 in 1992, and 178 in 1993) reflected a slightly higher proportion of females and a significantly higher proportion of whites than the general sample of employees. Those using outpatient mental health services were employed for roughly the same length of time and were of similar mean age to all employees in the sample. Mental health services users were significantly less likely to be married and had fewer numbers of dependents on average than the overall employee sample. Salaries were significantly higher among users in each year than for the overall sample.

Among mental health users, the mean number of outpatient mental health visits declined consistently and significantly each year: 11.5 mental health visits in 1990,10.8 mental health visits in $1991,9.1$ mental health visits in 1992, and 9.3 mental health visits in $1993(p<.05)$. The mean number of outpatient mental health visits in which network providers were used varied over the three years of the carve-out: in 1991, 79\% of all visits were provided by network providers; in 1992, 83\% were in network; and in 1993, 72\% were in network. The patient's mean mental health payment in each of the three years after the carve-out was significantly more than that paid in the year before the carve-out, although payments decreased slightly with each year after the carve-out $(\$ 277$ in 1990 , $\$ 318$ in 1991, \$313 in 1992, and \$301 in 1993). The company's mean outpatient mental health payment for mental health services users decreased significantly and consistently after the carve-out. The company paid an average of $\$ 646$ per mental health services user in 1990 , but $\$ 443$ in 1993 .

Table 1 presents the logit model (model 1) of the probability of mental health use. In the years after the carve-out, employees were 1.16 times more likely in the first year $(p<.1), 1.22$ times more likely in the second year $(p<.05)$, and 1.13 times more likely in the third year to use any mental health services as before the carve-out $(p<.24)$. Females were 1.30 times more likely than males to use any mental health services, and whites were almost two and a half times more likely than 
Table 1

Coefficients and Robust Standard Errors (in parentheses) for Models 1 through 4

\begin{tabular}{lcccccccc}
\hline Variable & \multicolumn{2}{c}{ Model 1 } & \multicolumn{2}{c}{ Model 2 } & \multicolumn{2}{c}{ Model 3 } & \multicolumn{2}{c}{ Model 4 } \\
\hline Carve-out 1991 & $.148^{*}$ & $(.087)$ & .006 & $(.103)$ & -.005 & $(.147)$ & -.147 & $(.125)$ \\
Carve-out 1992 & $.201^{* *}$ & $(.098)$ & -.054 & $(.105)$ & $-.271^{*}$ & $(.148)$ & $-.350^{* * *}(.126)$ \\
Carve-out 1993 & .118 & $(.101)$ & $-.183^{*}$ & $(.108)$ & $-.311^{* *}$ & $(.152)$ & $-.353^{* * *}(.134)$ \\
Female & $.243^{*}$ & $(.126)$ & -.098 & $(.107)$ & .118 & $(.155)$ & -.184 & $(.155)$ \\
White & $.891^{* * *}(.151)$ & $.359 * * *$ & $(.129)$ & $.447^{* *}$ & $(.188)$ & $.417^{* * *}(.155)$ \\
Age & $.187^{* * *}(.051)$ & .036 & $(.051)$ & .087 & $(.074)$ & .052 & $(.062)$ \\
Age squared & $-.002^{* * *}(.000)$ & -.001 & $(.001)$ & -.001 & $(.001)$ & -.001 & $(.001)$ \\
Years employed & -.013 & $(.008)$ & -.015 & $(.020)$ & -.001 & $(.030)$ & .012 & $(.024)$ \\
Number of dependents & $-.117^{* *}$ & $(.055)$ & $-.132 * * *$ & $(.049)$ & -.078 & $(.070)$ & $-.154^{* * *}(.060)$ \\
Salary & $.027 * *$ & $(.013)$ & $.069 * * *$ & $(.017)$ & $.116^{* * *}(.025)$ & $.063 * * *$ & $(.021)$ \\
Married & $-.279 * *$ & $(.139)$ & -.002 & $(1.07)$ & .037 & $(.154)$ & .030 & $(.128)$ \\
Constant & -6.74 & $(1.03)$ & 0.792 & $(1.03)$ & $2.52^{*}$ & $(1.48)$ & $4.86^{* * *}$ & $(1.25)$ \\
$R^{2}$ (overall) & & & & .09 & & .09 & & .11 \\
Probability $>\chi^{2}$ & & & & .000 & & .000 & & .000 \\
\hline
\end{tabular}

NOTE: Model 1 is a logit regression (generalized estimating equation with robust standard errors) of outpatient use of mental health (MH) services $(N=1,943)$. Models 2 through 4 are random effects generalized least squares regressions. Model 2 shows the natural logarithms (ln) of total MH use, Model 3 shows $\ln$ MH payments paid by the patient, and Model 4 shows $\ln$ MH payments paid by the employer. Models 2 through 4 are among users of MH services, any year, for continuously enrolled employees ( $n=167$ in 1990, 193 in 1991, 192 in 1992, and 178 in 1993).

${ }^{*} p<.1{ }^{* *} p<.01{ }^{* * *} p<.001$.

nonwhites to use mental health services. Being single increased the odds of using services over being married. Thus, those employees who were female, single, white, and who had slightly higher salaries than average were more likely to use any outpatient mental health services after the carve-out was implemented.

Also presented are the models for the level -in natural logarithms (ln) due to skewness-among mental health services users of total outpatient mental health services use (model 2), outpatient mental health service costs paid by the patient (model 3), and outpatient mental health service costs paid by the employer (model 4 ).

Model 2 estimates the level of outpatient mental health use (In of numbers of services) among users of outpatient mental health services. Being white and having a higher salary were significantly and positively related to level of use among users; the number of dependents was negatively associated. The carve-out did not reduce the level of mental health services use among users in the first two years of the carve-out, although the 1992 carve-out variable has a negative coefficient. Among the carve-out measures, only the carve-out variable for 1993 was significant at the $p<.1$ level; the coefficient indicates a $20 \%$ reduction in use. Overall $R^{2}$ is $.09(p<.001)$. The Hausman test indicated that the random effects were uncorrelated with the regressors, $\chi^{2}(8)=12.15$, probability $=.19$, which means the random effect model is an appropriate model to present.

Models 3 and 4 estimate outpatient mental health costs paid by the patient (model 3 ) and by the employer (model 4). In model 3, being white and having a higher salary are significant and positively associated with outpatient mental health payments over time. The carve-out measures for 1992 and 1993 (years 2 and 3 of the carve-out) are negative and significant, indicating that mental health 
payments paid by the patient were reduced by $31.1 \%$ in 1992 and $36.5 \%$ in 1993 , respectively, as compared with the pre-carve-out year. Overall $R^{2}$ is $.09(p<.001)$. The Hausman test again showed no systematic correlations (probability $=.14$ ).

Model 4 estimates the portion of mental health costs paid by the employer for users of outpatient mental health services. Having a higher salary and being white are positively and significantly associated with higher employer payments, and the coefficients on number of dependents are negative and significant. Again, the coefficients on the 1992 and 1993 carve-out variables are increasingly more negative and significant, indicating that mental health payments paid by the employer were reduced by $41.9 \%$ in 1992 and $42.3 \%$ relative to the pre-carve-out year. Overall $R^{2}$ is $.09(p<.001)$. The Hausman test again showed no systematic correlations in this model (probability $=.09$ ).

\section{Discussion}

The goals of managed behavioral health carve-outs include improving access to mental health services and controlling utilization. In this study of one employer, the introduction of the carve-out was simultaneously the introduction of a mental health benefit structure change, a PPO to a POS plan with a network of mental health providers, and an expansion of outpatient mental health coverage. In the first three years of the carve-out, the likelihood that employees received outpatient mental health services increased in part because of the carve-out. The increased probability of outpatient use was more evident in the first two years of the carve-out and less so in the third. White employees, those with higher salaries, and those with fewer dependents were also more likely to use mental health services after the carve-out than before.

However, once under care, the managed mental health carve-out model had a different effect, depending on the payer group of interest. Overall, the carve-out did not significantly affect the level of total outpatient mental health use over time among mental health services recipients. This means that for those under care, the fact that the carve-out existed did not influence the number of outpatient mental health services received over time. However, when mental health services payments are examined by source of payer (patient and employer), the time effects and role of the carve-out become more prominent. For the mental health services user, the carve-out decreased the level of patient and employer payments for outpatient mental health care in the second and third years of the carve-out but not significantly in the first. One may conclude that the administrative change from year 1 to year 2 for the providers did not affect costs to the users. The delay in impact on payments observed in the multivariate models may reflect the time required for employees to learn of the change in benefits or to convert to network providers for care. So, while the number of outpatient services was not affected by the carve-out, the costs to both employer and patient decreased as a result of this benefit change.

In summary, over the three years of experience with this carve-out, the sample of employees, albeit small, experienced an increased likelihood for outpatient mental health care and no change in the level of care once care was sought. Both the patient and the employer saw a decreasing level of expenditure for outpatient mental health services for those using services.

The generalizability of the study is limited by its focus on one private sector employer, although it was prototypical of medium-sized employers; one vendor, although it was typical of national managed behavioral health vendors; and one benefit plan. Although the PPO benefits and POS non-mental health benefits were standard for medium and large employers, the non-network mental health coinsurance amounts imposed here were higher than is typical. In addition, the sample size was very small, and inpatient use among this population was extremely limited-so much so that analysis of the potential cost offsets cited in other studies as a consequence of managed care cannot be addressed. As noted earlier, from this analysis we cannot estimate the extent of selection bias that may have resulted from differences among individuals who chose the HMO or the indemnity plan prior to the introduction of the carve-out. 


\section{Implications for Behavioral Health Services Delivery}

The study raises a number of questions about carve-outs in general. Research on the effects of mental health carve-outs on outpatient mental health care has revealed that carve-out strategies may lead to cost savings. However, if carve-outs are to be evaluated on the basis of their financial benefits, the likelihood, bearer, source, and timing of costs must be carefully examined. As noted here, although the carve-out did not affect the level of outpatient use over time once under care, costs to the employee and to the employer for outpatient mental health care decreased as a consequence of the carve-out over time.

If carve-outs are also to be assessed for their value or health benefit to the patient, then indicators of access and appropriateness must be included. This study indicates that access, if measured by an increased likelihood of receiving outpatient mental health services, may be improved by these arrangements. Although levels of use by those under care were not directly influenced by the carveout in this study, further examination of the patterns of use, types of therapy, and specialty of providers may indicate a differential effect of the carve-out mechanism for patients with varying needs. For instance, the carve-out may not affect the extent of use among low-level users but may more strongly influence the amount of use among high users. Primary care providers also deliver mental health care; it is not clear from this analysis if specialty mental health care received from carve-out network providers, perhaps at lower cost, replaced mental health care that had been provided in a primary care setting. This analysis does not address the issues of offsets and appropriateness of care, although we are examining the relationships between diagnoses and patterns of medical and mental health utilization in the sample.

Carve-outs continue to be a popular method for addressing the delivery and financing of mental health care in the private and public sector. Further monitoring and analysis are required to assure that the intent to provide accessible, high-quality, affordable mental health care to those in need is fulfilled.

\section{Acknowledgments}

This research was supported by National Institute of Mental Health grant MH 54135 and was performed at the University of Michigan, Ann Arbor.

\section{References}

1. Frank RG, McGuire TG: The economic functions of carve outs in managed care. American Journal of Managed Care 1998; 4:SP59-SP67.

2. Grazier KL, Eselius LL: Mental health carve-outs: Effects and implications. Medical Care Research and Review 1999; 56(2):37-59.

3. Caudron S: Carving out health-care savings. Personnel Journal 1995; April:38-48.

4. Goldman W, McCulloch J, Sturm R: Costs and use of mental health services before and after managed care. Health Affairs 1998; 17(3):40-52.

5. Callahan JJ, Shepard DS, Beinecke RH, et al.: Mental health/substance abuse treatment in managed care: The Massachusetts Medicaid experience. Health Affairs 1995; 14(3):173-184.

6. Frank RG, McGuire TG: Savings from a Medicaid carve-out for mental health and substance abuse services in Massachusetts. Psychiatric Services 1997; 48(9):1147-1152.

7. Ma CA, McGuire TG: Costs and incentives in a behavioral health carve-out. Health Affairs 1998; 17(2):53-69.

8. Christianson JB, Manning W, Lurie N, et al.: Utah's prepaid mental health plan: The first year. Health Affairs 1995; 14(3):160-172.

9. Sturm R, Goldman W, McCulloch J: Mental health and substance abuse parity: A case study of Ohio's state employee program. Journal of Mental Health Policy and Economics 1998; 1(3):129-134.

10. Dickey B, Norton EC, Normand S, et al.: Massachusetts Medicaid managed care health care reform: Treatment for the psychiatrically disabled. Advances in Health Economics and Health Research 1995; 5:99-116.

11. Dickey B, Normand ST, Norton EC, et al.: Managing the care of schizophrenia: Lessons from a 4-year Massachusetts Medicaid study. Archives of General Psychiatry 1996; 53:945-952.

12. Norton BC, Lindrooth RC, Dickey B: Cost-shifting in managed care. Working paper, 1996

13. Norton EC, Lindrooth RC, Dickey B: Cost-shifting in a mental health carve-out for the AFDC population. Health Care Financing Review 1997; 18(3):95-108. 
14. Stoner T, Manning W, Christianson J, et al.: Expenditures for mental health services in the Utah Prepaid Mental Health Plan. Health Care Fi* nancing Review 1997; 18(3):73-93.

15. Huskamp HA: The Impact of a Managed Behavioral Health Care Carve-Out and Benefit Expansion on Outpatient Spending for Mental Health and Substance Abuse Services. Unpublished manuscript, Harvard University, 1997.

16. Grazier KL, Scheffler RM, Bender-Kitz S, et al.: The effect of managed mental health care on use of outpatient mental health services in an employed population. Advances in Health Economics and Health Services Research 1993; 14:71-86.

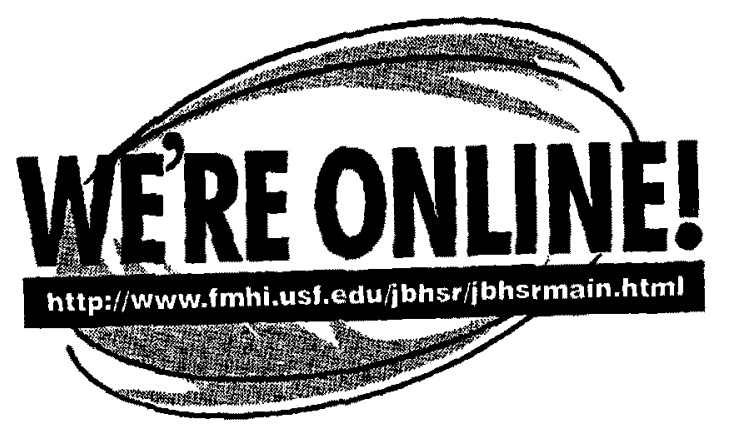

